\title{
Managing the Cultural Differences in Spain: When Talking about Interculturality Means Talking about Immigrant's Culture
}

\section{Pierangela Contini}

Mphil in Studi Etnici e Razziali (Trinity College - Dublino - Irlanda), Dottoranda in Antopologia Sociale (Università di Granada Spagna), Ricercatrice junior presso "Instituto de Migraciones" (Università di Granada - Spagna); pierangela.contini@gmail.com

\section{Antonia Olmos Alcaraz}

\author{
Dottore di Ricerca in Antropologia Sociale (Università di Granada - Spagna) \\ Professore Associato di Antropologia Sociale (Università di Granada - Spagna); antonia@ugr.es
}

\section{Paolo Contini}

Dottore di Ricerca in Demografia Storica e Sociale (Università degli studi di Bari) ed in Scienze Sociali (Pontificia Università San Tommaso d'Aquino - Angelicum - Roma), Professore a contratto di Sociologia del Mutamento Sociale (Università degli studi di Bari Aldo Moro) e professore invitato di Sociologia Generale (I.S.R.A. Facoltà Teologica Pugliese); paolo.contini@uniba.it

\section{Doi:10.5901/mjss.2016.v7n1s1p63}

\section{Abstract}

For many years, Spain is experimenting the phenomenon of immigration and implementing procedures normatively orientated according to the contexts, but sharing the main goal: integration. Those orientative processes have been first guided by multiculturalism and then evolving toward certain forms of interculturalism. On the base of this consideration it is necessary to deeply consider the theory debate on multi- and interculturalism, in order to consider the assumption and operational translations. Through the normative production and Third Sector discourses, emerge the trend of relating interculturalism to dynamics that are exclusively tied to the presence of migrant population in the Country. This essay is based on a qualitative research in which clearly emerged that, at least in Andalucia, but we can generalize to the whole country, the cultural managing process brought to interculturalism has been often difficult and did not help to definitely overcome assimilationist risks, typical above all of social operators at local level, for this reason we need to redefine the sense of "us".

Keywords: Inmigration, multiculturalism, interculturalism, Andalusia, Spain

\section{Introduzione ${ }^{1}$}

Da oltre un decennio la Spagna è divenuta terra di immigrazione.

Nonostante i dati concernenti i migranti abbiano subito variazioni significative al rialzo - a causa della condizione di crisi perdurante nella quale vive gran parte dei paesi del mondo - in Spagna sono presenti oltre cinque milioni di persone di nazionalità diversa da quella spagnola². Una quota rilevante di questi soggetti si ascrive alla categoria degli immigrati, nonostante siano nati in Spagna.

Intorno a questa popolazione e a quanti sono considerati - a diverso titolo - immigrati (nonostante, spesso, in possesso di nazionalità spagnola), si è diffusa la percezione della necessità di gestire la diversità, facendo rientrare nell'idea di diversità quella culturale (considerata come il "contenitore concettuale" per le differenze legate alla

\footnotetext{
1 II presente testo propone parte di alcune delle conclusioni del progetto di ricerca "Culturas de Convivência e Super diversidade" (PTDC/CS-SOC/101693/2008), finanziato dalla Fundação para a Ciência e a Tecnologia (Portogallo) e diretto dalla professoressa Beatriz Padilla con la partecipazione dell' Instituto de Migraciones dell' Università di Granada (Spagna). Ė inoltre parte della ricerca di dottorato di Pierangela Contini, svolta presso il Dipartimento di Antropologia Sociale e l' Instituto de Migraciones dell' Università di Granada. La sezione dedicata all'interculturalizzazione del sistema normativo spagnolo è parte della ricerca per la tesi di dottorato della professoressa Antonia Olmos Alcaraz (Olmos 2009). Si tratta di ricerche svolte principalmente in Spagna e in particolare in Andalusia, utilizzando una metodologia di ricerca qualitativa e tecniche etnografiche (interviste, osservazione partecipante, anlisi di testi normativi e dibattiti parlamentari, questi ultimi trascritti nella pagina web del Parlamento).

2 Dati statistici INE. Instituto Nacional de Estadística. http://www.ine.es (Ultimo accesso 10.09.2015).
} 
confessione religiosa e alla provenienza geografica).

Tra "nazionalità" e "immigrazione" non sussiste un nesso univoco, in quanto si tende a percepire come "immigrato" un soggetto prescindendo dal possesso della nazionalità: è la diversità culturale che lo rende "straniero". Se dunque, sul piano formale, un soggetto in possesso di nazionalità è da ritenersi "appartenente", dal punto di vista sostanziale "immigrazione" è una costruzione sociale che prescinde dal possesso di requisiti formali.

Questo tipo di approccio viene spesso estremizzato e si tende ad associare ad ogni immigrato, o ad ogni gruppo di immigrati, una cultura, che ciascuno porta con sé come se si trattasse di una "zavorra statica". Questo bagaglio è considerato più o meno problematico - o più o meno una risorsa - a seconda del modo in cui viene percepita la cultura del paese di provenienza.

Dal nostro punto di vista, il limite di questa prospettiva è insito nella caratteristica della staticità che si attribuisce alla cultura, e al non considerarla - correttamente, a nostro parere - come un processo passibile di mutamento, che evolve in stretta connessione con il contesto.

Sosteniamo, condividendo le posizioni di Barrett (2013), che la cultura di ogni gruppo sociale, di qualsiasi dimensione, comprende tra il resto, nazioni, gruppi etnici, città, quartieri, organizzazioni lavorative, gruppi di genere e generazionali. Da questa prospettiva deriva il fatto evidente che ciascuno possa appartenere contemporaneamente a più di una cultura intesa in questo modo, e si potrebbe addirittura pensare che non tutte convivono in maniera armoniosa.

La diversità del mondo è infinita, come ci suggerisce Boaventura De Sousa Santos (2011) e la diversità culturale è sempre esistita in quanto condizione umana.

Nel caso della Spagna, l'organizzazione in autonomie che hanno difeso le loro peculiarità culturali, come attesta la presenza di minoranze che hanno da sempre lottato per il riconoscimento dei propri diritti (come i baschi e i catalani), dimostra come questa "nuova diversità" - imputata ai nuovi flussi migratori - non sia così "nuova", così come non è "nuova" la necessità e la volontà di gestirla, secondo modi e forme diverse nel corso del tempo3.

Nonostante quanto appena detto, cioè che la Spagna non rappresenta in alcun modo un esempio di omogeneità culturale, è solo quando si è resa più evidente la presenza di gruppi immigrati che si è avviato un ampio dibattito sul tema ${ }^{4}$, nel quale i termini "interculturalità" o "multiculturalismo" sono stati usati in diversi ambiti e non sempre con le stesse intenzioni.

II fatto che non si stia sostenendo alcuna novità è asseverato da García e Granados (2002), i quali oltre un decennio fa già denunciavano l'intima relazione che si stabiliva tra i discorsi ed i disegni di azioni ed interventi interculturali da un lato, e la presenza di un tipo specifico di popolazione immigrante straniera nel territorio spagnolo dall'altro: "Il discorso interculturale si produce, dunque, con gli stranieri provenienti da altri paesi chiamati del Terzo Mondo"5 (García e Granados 2002: 4). Questa affermazione continua oggi ad avere senso e ad essere rilevante, insistendo sul fatto che parlare di interculturalità in Spagna vuol dire parlare di immigrazione e della "nuova" diversità culturale che l'immigrazione porta con sé.

Prima di continuare, è necessario ricordare alcune considerazioni espresse in lavori precedenti (García Castaño et al. 2011) circa le possibili declinazioni dei termini "interculturalità" e "multiculturalità". Innanzi tutto occorre, onde evitare confusione, specificare i contesti in cui si esprimono questi concetti:

II termine multiculturale si utilizza nella bibliografia anglosassone (Stati Uniti, Regno Unito, Australia) nella stessa maniera in cui in paesi europei e francofoni (Francia, Germania, Italia, Spagna ed in parte Canada) si utilizza e si preferisce il termine interculturale, dotandolo di un significato normativo, giacché sembra supporre l'interazione tra gruppi culturali che coabitano in uno stesso posto. II termine multiculturale in questi ultimi paesi ha visto ridurre la sua accezione ad un uso puramente descrittivo, riferendosi, come già abbiamo detto, ad una supposta coesistenza di diverse culture in uno stesso spazio (García Castaño et al. 2011: 6).

Negli ultimi anni la relazione tra interculturalismo e multiculturalismo è stata oggetto di numerosi dibattiti accademici circa il loro impiego, ritenuto, a volte, inappropriato.

Se da un lato per alcuni autori questi concetti si associano a due focus molto diversi, per altri, come per esempio

\footnotetext{
${ }^{3}$ Ricordiamo che durante il periodo franchista la gestione della diversità ha assunto toni assimilazionisti rispetto, per esempio, all'uso di lingue diverse dal castigliano.

${ }^{4}$ In Spagna si inizia a parlare di immigrazione a metà degli anni Ottanta, a partire dall'emanazione della prima "Ley de Extranjería" (Ley Orgánica 5/1985), che fu interpretata come un "requisito" per l'ingresso del paese nell'allora Comunità Economica Europea. Da allora si inizia un proceso di "politicizzazione del fenomeno migratorio" (López, 2005) che favorirà la costruzione sociale dell'immigrazione "come problema" (Santamaría, 2002; Olmos, 2007), qualcosa che risponde più al momento politico del paese che al numero di stranieri presenti in quel momento (Olmos, 2008, 2010).

${ }^{5}$ Specifichiamo che sono nostre tutte le traduzioni delle citazioni di testi la cui lingua originale non è l'italiana.
} 
Meer e Modood (2012), l'interculturalismo semplicemente ritorna a enfatizzare alcuni punti chiave già inclusi nelle versioni più recenti del multiculturalismo.

Barrett avverte che un'altra complicazione è dovuta al fatto che:

\begin{abstract}
In Canada il termine interculturalismo è utilizzato per definire un modello specifico di gestione della diversità culturale in Québec, che è stato articolato esplicitamente in opposizione al multiculturalismo canadese (Bouchard 2011), e che però differisce dal modello europeo di interculturalismo. In più alcuni autori (come per esempio Kymlicka 2012) hanno segnalato che nonostante $i$ due focus non sono chiaramente diversi, può darsi un vantaggio strategico nel preferire l'uso del termine "interculturalismo" dovuto al fatto che il termine "multiculturalismo" è diventato politicamente corrotto negli ultimi decenni (Barrett 2013: 15).
\end{abstract}

Un'ulteriore difficoltà è dovuta all'uso spregiudicato di questi termini in diversi ambiti: accademico, politico, pedagogico e massmediatico, dove non sempre si esplicita quello che si intende per interculturale o multiculturale, generando così importanti confusioni tra cui l'errore di presentare con lo stesso termine, modalità di gestione della diversità assai difformi l'una dall'altra 6 .

È bene ricordare che la condizione normale di tutti i gruppi sociali è la diversità, per questa ragione sarebbe necessario fare riferimento a "società o gruppi diversi" invece che a "società o gruppi con molteplici culture".

Nell'ambito delle scienze sociali il dibattito concettuale sul significato del termine cultura e delle sue declinazioni inter e multi, non è per niente nuovo.

Nonostante non sia questo l'oggetto del presente lavoro, riteniamo che alcuni chiarimenti siano necessari. Carlo Giménez, in questo senso, pone in relazione il multi e l'interculturale con il pluralismo culturale?:

... entrambe le idee (multiculturale ed interculturale) hanno in comune i pilastri dell'edificio proposto dal pluralismo culturale (valutazione positiva della diversità, critica delle proposte di perdita o differenza culturale, uguaglianza e non discriminazione per ragioni di differenziazione etnoculturale delle persone, rispetto della diversità, etc.), ed è per questo che riteniamo più corretto - e chiarificatore - considerare il multiculturalismo e l'interculturalità come concrezioni successive del paradigma pluralista (Giménez 2003).

Utilizzando il termine multiculturalità si farebbe quindi riferimento al piano "fattivo" (realtà sociale in cui esiste la diversità culturale, linguistica, religiosa, etc.) mentre il termine multiculturalismo si collocherebbe in un piano normativo, riferendosi al riconoscimento attivo, sociale ed istituzionale della differenza, e che è la base di modelli politici e/o etici.

La stessa distinzione è riferibile ai termini interculturalità ed interculturalismo, in questo caso però, lo stesso autore evidenzia qualche sfumatura:

La prospettiva interculturale è diventata necessaria e fino ad ora, tutto sembra indicare che lo sarà in futuro, a causa delle limitazioni, degli errori e dei fraintendimenti determinati dal multiculturalismo (...) Le critiche citate hanno come focus comune l'insufficienza dell'idea multiculturale come progetto di coesione sociale della comunità politica dove si collocano le differenze (Giménez 2003: 12).

Se da una parte Giménez lo propone come un superamento del multiculturalismo, per Javier De Lucas l'interculturalismo suppone una modalità di gestione della multiculturalità:

... l'interculturalismo, così come ha spiegato magistralmente il filosofo messicano Luis Villatoro (1998), è solo una maniera di gestire la multiculturalità, che cerca di essere rispettosa nei confronti della diversità proponendo un reciproco riconoscimento delle due o più culture in co-presenza ${ }^{8}$, e che esige la volontà di mutua conoscenza di queste e non l'intento di sopprimere le caratteristiche di quelle più deboli in ragione dei pregiudizi che abbia di queste la (cultura)

\footnotetext{
${ }^{6}$ Per un approfondimento sulle differenze tra i significati di questi due termini si rimanda a García Castaño et al. 2011, e Garcia Castaño et al. 2012, in cui si presentano, a livello elementare, "multiculturalità" e "interculturalità" ed $i$ termini ad essi associati. L'aspetto centrale è sempre rappresentato dal concetto di cultura che sottende all'uso di questi termini.

${ }^{7}$ Diversi autori hanno interpretato la relazione tra il piano normativo espresso dal multiculturalismo ed il pluralismo culturale, come una relazione logica:

Inizialmente, nonostante il multiculturalismo non sia stato un concetto dall'interpretazione univoca, lo si considerava come una variante dell'espressione "pluralismo culturale", espressione che definisce le società plurali nelle quali convivono, in maniera armonizzata, differenze etniche, culturali, religiose, linguistiche, etc. Questa locuzione esprime in certo modo l'opportuna tolleranza verso la diversità culturale, foriera di un atteggiamento rispettoso nei confronti delle minoranze, alla base di qualunque tentativo di promozione della democrazia sociale (Andreo Tudela 2004: 13).

$8 / \mid$ corsivo è nostro ed esprime una certa difficoltà nel pensare a "due culture che vivono insieme".
} 


\section{dominante (De Lucas 2002: 21).}

II mondo accademico di tradizione altra da quella occidentale critica l'uso di questi concetti: l'interculturalismo descriverebbe una risposta critica al multiculturalismo. Per Meer e Modood (2012) - e non solo per loro, in realtà - il multiculturalismo non rappresenta più un concetto pienamente convincente, e da ciò deriverebbe la necessità di integrarlo nel più ampio e convincente concetto di interculturalismo.

Ma parlare della diversità culturale in questi termini suppone la convivenza in uno stesso territorio di varie culture, di diverse provenienze. Un'interpretazione di questo genere ha come sottesa l'idea di "frontiera": è come se ogni gruppo appartenesse ad una cultura ed ogni cultura ad un gruppo.

In questo lavoro non pretendiamo approfondire su questo dibattito concettuale, ma piuttosto sull'uso dei due termini in diversi ambiti. Seguendo quest' obbiettivo, vedremo a che punto si trova il dibattito sull'interculturalismo ed il multiculturalismo in Spagna suggerendo, in seguito, un'analisi critica dei termini e dei significati attribuiti ${ }^{9}$.

Ci concentreremo principalmente su due aspetti: prima di tutto sulla produzione normativa relativa al fenomeno delle migrazioni (o più in generale, al fenomeno della presenza straniera) dato che, come cercheremo di dimostrare, il dibattito sull'Intercultura è sorto in stretta relazione alla crescita di questa popolazione.

Successivamente osserveremo come l'interculturalità, per la stessa ragione, sia un concetto essenziale nei discorsi di alcune ONG attive sul territorio.

Infine, utilizzando i concetti di "interculturalismo funzionale" e di "interculturalismo critico" che ci vengono offerti dalla prospettiva Latinoamericana, ed in concreto da Catherine Walsh (2010) esporremo la nostra posizione in merito alla tendenza delle istituzioni (governative e non) di porre in stretta relazione l'interculturalismo con il fenomeno migratorio e la "questione culturale" che ne deriva.

\section{Come si è Interculturalizzata la Normativa Spagnola? II Caso dell'Andalusia}

Sul finire degli anni Novanta dello scorso secolo, nelle politiche e nella produzione normativa spagnola iniziò ad introdursi una prospettiva di tipo interculturale. Fu in quel periodo che, soprattutto nell'ambito educativo, si iniziò a considerare la necessità di integrare la popolazione migrante 0 straniera. Evidentemente la presenza straniera era una caratteristica della Spagna anche prima di quel periodo, così come - anche prima - si erano poste in essere delle strategie di integrazione, ma è solo dalla fine del XX secolo che il fenomeno dell'integrazione si è affrontato in termini di interculturalità fino ad arrivare a fare riferimento a "un modello di integrazione interculturale" (Rodríguez García 2011) (rispetto alla relazione tra interculturalismo e migrazione si vedano i testi di: Diaz y Alonso 1998, Cárdenas Rodríguez 2002, Leunda 2008, Rodríguez García, 2011).

Per una migliore comprensione del termine "interculturale" in Spagna, occorre esaminare le normative che disciplinano l'integrazione delle popolazioni migranti e straniere nel Paese, ed è per questo che è opportuno considerare questi strumenti giuridici applicati a livello regionale, in quanto le diverse comunità autonome hanno, nell'esercizio della propria autonomia, competenze specifiche in ragione delle quali possono declinare i propri piani e i propri progetti soprattutto per quanto concerne le politiche di integrazione. Tuttavia, nonostante le specificità territoriali è ragionevole ritenere i risultati delle nostre analisi come generalizzabili all'intera Spagna, poiché stante la declinazione locale, le normative derivano comunque dalla Ley de Extranjeria, legge generica dello Stato in materia di Immigrazione.

Nell'ambito delle normative andaluse, vanno segnalati i Planes Integrales para Inmigración (Piani Integrali per l'immigrazione) (nelle loro diverse edizioni: I per gli anni 2001 - 2001, II per il 2006 - 2009 e III per il 2014 - 2016), che rispondono alle indicazioni che la Ley de Extranjeria stabilisce a proposito dell'integrazione; ed il Plan de Atención al Alumnado Inmigrante (Piano di Attenzione agli Alunni Immigrati) del 2001 - poi chiamato Plan para la Educación Intercultural ${ }^{10}$ (Piano per l'Educazione Interculturale).

\footnotetext{
${ }_{9}^{9}$ Faremo frequentemente riferimento al territorio andaluso, in quanto uno dei nostri contesti geografici di ricerca, e che consideriamo esemplificativo per l'analisi dei fenomeni studiati.

10 II Plan para la Atención al Alumnado Inmigrante è di poco precedente al I Plan Integral para la Inmigración en Andalucía in cui sarà successivamente integrato, principalmente per offrire un supporto istituzionale alle Aule Temporali di Adattazione Linguistica (ATAL) già in funzione in Andalusia dall'anno 1997. II Plan de Atención al Alumnado Inmigrante fu promosso dalla Consejería de Educación y Ciencia della Giunta d'Andalusia, come il risultato di un processo di consulenza del professorato, altri agenti educativi e sociali ed esperti universitari che lavoravano questioni relative all'immigrazione. La Giunta d'Andalusia è l'organo esecutivo della comunità autonoma andalusa, che si articola in diversi dicasteri, tra i quali la Consejería de Educación y Ciencia citata nel presente testo. L'integrazione di questo Piano nel I Plan Integral para la Inmigración en Andalucia - nelle sue diverse versioni rinnovate - fu motivata dall'interesse esistente a livello istituzionale di centralizzare nella Consejería de Gobernación (organo regionale equivalente al Ministero dell'Interno),
} 
Ma di che genere di interculturalità parlano questi atti?

Se guardiamo al modo in cui il termine" interculturalità" è utilizzato genericamente nei programmi integrali dell'immigrazione, scopriamo che nel I Piano si considerava l'interculturalità come uno dei principi fondanti e veniva descritta come un processo, che seguendo i dettami della Carta dei Diritti Umani, era volto all' interazione tra popolazioni immigrate e popolazione autoctona, nel rispetto delle culture di tutti:

Interculturalità: la pluralità culturale che il fenomeno dell'immigrazione comporta può contribuire ad un processo reale e dinamico di interazione tra la società di accoglienza e la popolazione immigrata, cosi come con il contesto ecologico e con gli spazi sociali dove tale processo si produce, salvaguardando il rispetto per le singole culture e le loro peculiarità, seguendo il dettato della dichiarazione dei Diritti Umani (I Plan Integral para la Inmigración en Andalucía 2001: 65).

Ancora una volta si propone un modello di immigrazione foriero di culture proprie e latore di "peculiarità". I primi passi delle politiche di integrazione della popolazione migrante guardano all'interculturalità come ad un obiettivo da raggiungere collettivamente per ottenere la piena integrazione tra popolazioni culturalmente diverse e relazioni armoniche con la cultura della maggioranza (autoctona).

Nel II Plan Integral para la Inmigración en Andalucía l'interculturalità è ancora un principio base. Analizzandone la redazione è facile vedere la similitudine tra questo Piano ed il primo, ad eccezione del fatto che questa volta l'interculturalità si considera come una prerogativa già integrata nel sistema politico e istituzionale andaluso:

Interculturalità: la pluralità sarà considerata come uno dei vantaggi offerti dal modello di integrazione sul quale punta la Junta de Andalucia, favorendo l'integrazione nella società andalusa. (II Plan Integral para la Inmigración en Andalucia 2006:146).

Dunque possiamo rilevare un cambiamento di prospettiva rispetto al Piano precedente: nel primo si faceva riferimento all'interculturalità come a un vantaggio utile da adottare nei processi di integrazione, nel secondo si da già per incorporata nei processi stessi. Di fatto si evidenzia la consapevolezza dell'amministrazione andalusa di avere adottato, tra le due edizioni del Plan Integral para la Inmigraciòn en Andalucía, delle politiche pubbliche interculturali ${ }^{11}$.

Per quanto attiene al III Plan Integral para la Inmigración en Andalucia, la sua entrata in vigore era prevista nel 2009 (rimanendo valido fino al 2013), tuttavia diverse ragioni hanno post-posto l'approvazione del Piano che è stato approvato solo nel 2014. I commenti al Piano sottolineano, quale elemento di novità, il voler "mantenere i risultati raggiunti", in specie l' "efficace gestione della diversità culturale ${ }^{12}$, offrendo tra l'altro una rinnovata interpretazione dell'interculturalismo:

"Interculturalismo" è la possibilità di sviluppare molteplici e complesse identità che permettano di collaborare in vista del bene comune. Bene che, necessariamente, deve permettere il libero sviluppo della personalità di ogni essere umano. Si tratta di una sfida alla società: occorre convertire i nuovi cittadini e cittadine in soggetti di pieno diritto, consentendo loro, contemporaneamente, di mantenere le caratteristiche culturali peculiari della propria identità. $\dot{E}$ necessario valutare la diversità definendo un nuovo spazio di negoziazione in cui collaborare alla costruzione di una grande cultura comune, caratterizzata dalla volontà di convivenza, non solo pacifica, ma anche foriera di pari condizioni di dignità e di accesso alle opportunità (III Plan Integral para la Inmigración en Andalucía 2014: 82).

In questo testo scompare il riferimento agli "immigrati" o agli "stranieri", preferendo un costruttivo "nuovi cittadini".

tutte le attuazioni che avevano a che fare con la gestione del fenomeno migratorio. La lettura che possiamo fare a riguardo ci porta a ritenere che in quel contesto specifico la Giunta d'Andalusia volesse distinguersi da chi gestiva questa realtà solo dal punto di vista del lavoro - cosa che presuppone uno stereotipo di immigrato inteso esclusivamente come lavoratore - o solo dalle questioni sociali -che presuppone, essenzialmente, una nozione della popolazione immigrata come insieme di soggetti aventi bisogno di attenzione sociale. Attualmente questi principi sono cambiati e la gestione delle politiche migratorie in Andalusia è attuata dalla Consejería de Empleo. Per approfondimenti sul tema si veda Agrela (2006), García e Olmos (2010) e Martínez Chicón (2012).

11 II II Plan Integral para la Inmigración en Andalucía, rispetto alla versione precedente, introduce alcune piccole modifiche sui principi che lo devono guidare. Appaiono temini quali adattabilità, cooperazione, integralità e trasversalità per sostituire uguaglianza e globalità.

Questo dimostra la volontà da parte del Governo regionale di adattare le politiche a zone concrete (adattabilità); lavorare assieme alle amministrazioni locali ed allo Stato (cooperazione); contemplare aspetti di promozione ed attenzione all'integrazione (integralità) e di considerare l'importanza di agire in maniera trasversale su tutte le questioni che riguardano l'immigrazione: educazione, sanità, lavoro, cultura (trasversalità) (II Plan integral para la Inmigración en Andalucía 2006: 145). Per approfondimenti su questo tema, consigliamo di consultare i lavori di García e Olmos (2010), Andreo Tudela e Santa Cruz (2011).

${ }_{12}$ Dalla presentazione ufficiale del III Plan Integral para la Inmigración en Andalucía, riportata al sito http://www.juntadeandalucia.es/ organismos/justiciaeinterior/areas/politicas-migratorias/planes-inmigracion.html (Ultimo accesso 17/08/2015). 
Un'altra novità è insita nel fatto che per la prima volta si fa riferimento ad "una grande cultura comune".

Nonostante i cambiamenti sostanziali rispetto agli strumenti precedenti, è ancora troppo presto per valutare le implicazioni pratiche che questi potranno avere e che tra l'altro dovranno trovare concretezza in un contesto di crisi economica perdurante, assai diverso da quanto accaduto in precedenza.

Tuttavia, come si accennava prima, l'ambito in cui meglio si manifestano le dinamiche interculturali dell'attività normativa, è l'educativo. In Andalusia quella che viene definita come "educazione interculturale"13 si propone come la soluzione alternativa, che parte dalla scuola, per ottenere l'integrazione della popolazione immigrata.

Già nel 1999 nella Ley de Solidaridad en la Educación ${ }^{14}$ (Legge di Solidarietà nell'Educazione) si sottolineava, al secondo articolo, come uno dei suoi obiettivi sarebbe stato quello di: "...potenziare il valore dell'interculturalità, integrando nell'educazione la ricchezza presupposta dalla conoscenza e dal rispetto per la cultura dei gruppi minoritari e sviluppare attitudini di comunicazione e rispetto tra i membri della comunità educativa, indipendentemente dalle loro capacità personali e dalla loro condizione socioculturale"15.

Questi principi saranno poi accolti dal Plan para la Atención Educativa del Alumnado Inmigrante (Piano per l'attenzione Educativa per gli studenti immigrati) elaborato nel 2001, in cui si specifica in maniera puntuale in cosa consista la prospettiva interculturale e quali siano le azioni concrete volte ad implementarla:

Il concetto chiave della prospettiva interculturale è l'idea di "interazione" (...) che è possibile solo se si instaura un processo che vede coinvolti sia gli attori autoctoni che gli immigrati, e che prevede una negoziazione dei significati, resi oggetto di interscambio e di valutazione critica. L'interculturalità supera la prospettiva multiculturale perché, partendo dal riconoscimento della diversità culturale come dato di fatto, vede l'interazione culturale come un "fatto educativo", in sé stesso. L'interculturalità obbliga a pensare alla relazione culturale inserita in un progetto educativo, ma anche in un progetto sociale, che possa rendere possibile la parità dei diritti e delle opportunità tra esseri umani che convivono in una determinata società (Plan para la Atenciòn Educativa del Alumnado Inmigrante 2001: 8-9).

Riconoscimento reciproco, uguaglianza, scambio, prospettiva critica e creazione di nuove realtà: sembrano essere questi i principi che appaiono in tutti gli strumenti normativi delle Comunità Autonome, che parlano di interculturalità. In un certo senso, si evidenziano le differenze tra il paradigma multiculturale - in quanto mera presenza della diversità - e quello interculturale - in quanto interrelazione e interscambio - e la desiderabilità di quest'ultimo. Ciò nonostante, occorre considerare che questa "interculturalizzazione" delle normative e politiche andaluse ha avuto uno sviluppo concreto coerente a quanto espresso a livello normativo. Per verificare ciò è sufficiente fare un esercizio comparativo tra quello che si propone nella normativa e quello che realmente si declina nella pratica. Ė inoltre opportuno osservare ciò che si intende per interculturalità in questi documenti e quali siano le questioni a cui si fa riferimento a livello di dibattito parlamentare. Vediamolo con più attenzione.

Rispetto alla relazione tra quello che si pianifica nelle norme e quello che si esegue con le politiche, evidenziamo, a titolo di esempio, che quando nel Plan para la Atención Educativa del Alumnado Inmigrante (Piano per l'Attenzione all'alunnato immigrante) si afferma che l'interculturalità sarà possibile solo "se si stabilisce un processo in cui tutti possano apportare contributi e in cui questi contributi siano soggetto di interscambio e valutazione critica" (2001: 8), ci si potrebbe aspettare che per ottenere questo modello di interculturalità, tutti i gruppi presenti nella scuola possano realizzare questo scambio in condizioni di uguaglianza. Nonostante queste dichiarazioni di intenti, notiamo come la principale (se non l'unica) azione che questo Piano ha mantenuto e sviluppato durante i quasi 15 anni in cui è in vigore, è rappresentata dalle lezioni di spagnolo per determinati alunni stranieri attraverso le Aule Temporali di Adattamento Linguistico (Aulas Temporales de Adaptación Linguistica), conosciute come ATAL. Per questo è opportuno chiedersi quale sia l'apporto che danno fattivamente i gruppi non ispano parlanti all'auspicato interscambio 0 , in altri termini, che cosa connoti come interculturale il fatto che altri imparino la lingua del paese ospitante.

\footnotetext{
${ }^{13}$ Per un'analisi approfondita dei concetti e della realtà dell'educazione interculturale in Spagna, si vedano i testi di: Garcia Castaño et al., (1999); García Castaño e Granados Martinez (2002); García Castaño et al., (2007), García-Cano Torrico et al. (2009); Olmos Alcaraz (2004) o Pulido Moyano (2005).

${ }^{14}$ Legge Andalusa 9/1999, del 18 novembre, Solidaridad en la Educación (Solidarietà nell'educazione).

${ }^{15}$ Ciononostante esiste un precedente nel 1997, il Piano integrale per la Comunità Gitana di Andalusia (Plan Integral para la Comunidad Gitana de Andalucía) (BOJA del 20 febbraio del 1997), con il quale si stabilisce la necessità di un focus interculturale nelle scuole con la promozione di attività di convivenza pluriculturale (attuazione 5), incentivazione dello studio della cultura gitana nella formazione dei docenti e corsi di formazione al rispetto (attuazione 7, 8), al fine di promuovere una inclusione positiva della cultura gitana nella proposta didattica (attuazione 10).
} 
II dibattito sull'interculturalità a livello parlamentare ${ }^{16}$ non si è occupato della diversità culturale nelle sue varie declinazioni, ma si è focalizzato sul tema dell'immigrazione, quasi a considerarla l'unica origine di diversità nella società. La Comisión de Igualdad y Bienestar Social scrive:

... proponiamo un'iniziativa, non una proposta di legge che ha come titolo (...) la mediazione interculturale dei servizi sociali (...) che non ha altro obiettivo che migliorare la vita della popolazione immigrata (Parlamentare del Grupo Socialista. Comisión de Igualdad y Bienestar Social, 04/09/2009)

Ed anche sul piano scolastico la lettura è assai simile:

"abbiamo aumentato, anno dopo anno, il numero di professori destinati agli immigrati" (Consejera de Educación, Seduta della Camera, 14/06/2006).

Sempre al 2006 risale una delle dichiarazioni più esplicite in questo senso:

\begin{abstract}
... l'interculturalità, la tolleranza ed il rispetto sono fattori fondamentali per una convivenza armonica, ma lo è anche l'integrazione, e integrazione significa che partecipino (gli immigrati) dei valori culturali della libertà, democrazia, uguaglianza di diritti e solidarietà che caratterizzano la nostra società (Parlamentare del Grupo Popular, Seduta della Camera, 2/03/2006).
\end{abstract}

Questo genere di espressioni mostra come l'interculturalità sia declinata "a senso unico": migliorerà la vita degli immigrati, nella misura in cui loro (solo loro!) partecipino dei valori dominanti della società di accoglienza. Della "nostra società".

\title{
3. L'interculturalità nelle Organizzazioni non Spagnole
}

Come abbiamo visto, il processo di interculturalizzazione, associato all'immigrazione ed alla gestione della diversità culturale, è penetrato in Spagna nella normativa e nella politica, sia nel caso generale dei piani di integrazione, sia in maniera più specifica, in ambiti come l'educazione - mediante i piani e le normative emanate dalle diverse Comunità Autonome - come nel citato caso dell'Andalusia. Ed anche nel dibattito parlamentare esiste la preoccupazione per la gestione della diversità culturale.

Occorre chiedersi che cosa succeda con le Organizzazioni non Governative e come si relazioni il Terzo Settore con il tema dell'interculturalità: come lo percepisce e lo definisce? Come intende implementarlo ed in che area di intervento lo colloca? Che cosa si intende con la parola interculturalità?

Anche in questo caso osserviamo che il dibattito sull'interculturalità e le prassi ad essa connesse quasi sempre si riferiscono a temi migratori, ed il Terzo Settore non è un'eccezione ${ }^{17}$.

Le associazioni che hanno tra gli interessi prioritari l'interculturalità, si pongono come obiettivo specifico l'attenzione alle popolazioni immigrate e, concretamente, la loro integrazione nella società. Nessuna di loro opera in ambito nazionale, e la necessità di una dimensione locale diventa comprensibile se si associa l'inserimento-inclusioneintegrazione sociale e lavorativa alla popolazione immigrante.

A mero titolo esemplificativo, ci soffermeremo su tre associazioni, regionale la prima, internazionale le altre due, che adottano approcci e stili diversi, e come tali possono essere considerate rappresentative del modus operandi in questo senso delle ONG presenti sul territorio (spagnolo in generale, andaluso in particolare).

Un esempio nel nostro contesto geografico di ricerca lo offre l'associazione di volontariato "Andalusia Accoge" (Andalusia Accoglie) che, coordinata a livello regionale, opera sul territorio organizzandosi in una rete composta da 9 associazioni locali. Andalucia Acoge è sorta nel 1991 "per dare una risposta più efficace e globale all'incipiente fenomeno dell'immigrazione". II suo obiettivo generale è favorire l'integrazione degli immigrati nella società di accoglienza e promuovere l'interculturalità attraverso progetti come La Scuola Interculturale del Volontariato, pensata per favorire la partecipazione delle persone immigrate nella società di arrivo.

La definizione che l'associazione propone di questo concetto, si impernia intorno alla relazione positiva di comunicazione tra persone di diverse culture che coesistono in uno stesso contesto geografico.

Anche alcune delle associazioni di aiuto pianificano in maniera isolata la questione interculturale ancora una volta

${ }^{16}$ Per un'analisi approfondita sul discorso parlamentare in Andalusia su interculturalità e immigrazione, si vedano i testi di Olmos Alcaraz (2007, 2009). Per un'analisi a livello statale, si può consultare la tesi di dottorato di Márquez Lepe (2006).

${ }^{17}$ Come già affermato, l'interculturalità è un tema che generalmente si affronta con questioni concernenti l'inserimento, l'inclusione e l'integrazione sociale e lavorativa della popolazione immigrata nella società dominante. II compito di raggiungere questi obiettivi è affidato alle ONG a cui i governi (tanto il governo centrale come quelli regionali) fanno riferimento mediante il sostegno economico a progetti di inserimento lavorativo, formazione linguistica, etc. 
pensando all'appoggio alle popolazioni immigrate e con un approccio più o meno paternalista.

Per Caritas l'interculturalità è strettamente connessa con la prospettiva solidaristica. Caritas non ha bisogno di presentazioni, né a proposito degli ambiti di intervento, né in relazione all'orientamento religioso, nonostante sia notevole lo sforzo di discostarsi dagli stereotipi clericali (Dietz et al. 2000). In un documento pubblicato nell'anno 2000, "Caritas e Immigrazione" si legge:

\begin{abstract}
...il supporto ed il servizio solidale rappresentano gli interventi giornalieri: i passi avanti ed indietro, "le gioie, le fatiche e le speranze" vissute dai nostri collaboratori e dagli stessi immigrati nelle lezioni di lingua e cultura, nelle consulenze, mediazioni, nella ricerca dei documenti, lavoro o alloggio, di aiuto presso le mense nelle scuole per i bambini. In tutto ciò affiorano la difficoltà e l'arricchimento con l'interscambio di modi di vedere e di sentire, impensabili fino a pochi anni fa, quando non conoscevamo nemmeno la parola "interculturalità" (Herrera 2000: 13).
\end{abstract}

Più recentemente sulla pagina web di Caritas é apparso un documento dal titolo "Construir la convivencia intercultural" dove si mette in relazione l'interculturalità con una convivenza consolidata e costruita, nella quale, citando Papa Francesco, "passare dalla cultura del rifiuto alla cultura dell'incontro"18

Un altro ente di volontariato assai noto a livello internazionale è la Croce Rossa. Storicamente conosciuta come un'associazione di solidarietà, nel 2006 pubblica un documento intitolato Pistas metodológicas para la sensibilización cultural (Piste metodologiche per la sensibilizzazione interculturale) (Cruz Roja 2006) dove chiarisce, sempre nell'ambito del discorso migratorio, che dal proprio punto di vista, la prospettiva interculturale suppone, paragonata ad altre visioni come l'assimilazione o l'emarginazione:

- Un elemento attivo di ricerca dell'incontro culturale in un contesto di uguaglianza

- Una valutazione positiva dell'incontro.

- Una visione critica delle culture che cerca di evitare sia il paternalismo, sia la concezione gerarchica della cultura.

Dunque, quando non ci si orienta verso meccanismi di inserimento o di integrazione, il problema torna ad essere la cultura e per la Croce Rossa la questione in concreto è il paternalismo e la concezione gerarchica della cultura medesima.

Come già detto, sono esclusivamente le associazioni il cui obiettivo principale è l'attenzione all'immigrato, quelle che focalizzano le loro cure sul tema dell'interculturalismo, mentre le altre percepiscono l'interculturalità solo come una delle possibili aree di lavoro.

Dunque anche per quanto riguarda il Terzo Settore spagnolo, si registra come sia le pratiche che il dibattito interculturali fanno sempre riferimento all'integrazione, al supporto e all'inserimento degli immigranti.

La proposta è quella di un dialogo la cui pretesa è quella dell'orizzontalità e dell'interculturalità, che prevede lo scambio tra "culture", spesso secondo la più essenzializzata delle accezioni che ne evidenzia l'aspetto folclorico, quanto in gioco c'è la cultura delle minoranza.

Tutte queste proposte ovviano spesso le condizioni di disuguaglianza in cui vivono i settori della popolazione a cui si rivolgono. In questi termini l'orizzontalità e l'interculturalità che auspicano possono risultare fittizie e nascondere categorie coloniali (che riproducono le stesse categorie egemoniche di potere), ossia atteggiamenti "buonisti" che, in nome di un'apparente mediazione, ottengono come risultato ultimo l'assimilazione e, sostanzialmente, l'elisione delle specificità culturali delle minoranze, pratica già risultata non efficace in altri contesti19.

\title{
4. Alcune Riflessioni Conclusive
}

Come si può definire il tipo di interculturalità che abbiamo presentato? Catherine Walsh, riprendendo Fidel Tubino Arias, propone l'idea di "interculturalismo funzionale" diverso dall' "interculturalismo critico" (Walsh 2010) 20. Questa differenziazione può essere utile nell'interpretazione del processo d'interculturalizzazione in Spagna manifestatosi soprattutto attraverso del Terzo Settore.

L'interculturalità funzionale si basa sul riconoscimento della diversità e delle differenze culturali ed ha come

${ }^{18} \mathrm{http}: / /$ www.caritas.es/coriacaceres/noticias_tags_noticialnfo.aspx?ld=7942 (Ultimo accesso 18.11.2015).

19 II modello "assimilazionista" è stato sperimentato in Francia negli anni Ottanta del secolo scorso, e si veda a tale proposito Zanfrini (2004).

${ }_{20}^{2}$ Per "interculturalismo critico" intendiamo, con Fidel Tubino Arias, quell'approccio che mira a destrutturare le cause dell'asimmetria culturale: si tratta dunque di una modalità di confronto con le diversità assai distante dal rischio assimilazionista, per tanto molto diverso dallinterculturalismo funzionale (o neo-liberale), allo stato, dominante. A tale proposito di veda Tubino (2004). 
obiettivo l'inclusione delle diversità nella struttura sociale dello Stato Nazione. In questo modo l'interculturalità, finalizzata alla promozione della tolleranza, del dialogo e della convivenza, è "funzionale" al sistema esistente, poiché non incide sulle cause dell'asimmetria, della disuguaglianza sociale e culturale, né tanto meno mette in discussione le "regole del gioco", per questo è perfettamente compatibile con la logica del modello neo-liberale esistente (Tubino 2005, in Walsh 2010). Questa logica apparentemente promuove la differenza, la riconosce, però si adopera perché sia integrata nell'ordine sociale nazionale. Per Walsh:

\begin{abstract}
...ll riconoscimento ed il rispetto della diversità culturale costituiscono una nuova strategia di egemonia, che mira non alla creazione di società più giuste ed ugualitarie, ma al controllo del conflitto etnico ed alla conservazione della stabilità sociale con l'obiettivo di spingere gli imperativi economici del modello (neoliberale) di accumulazione capitalista, "includendo" i gruppi storicamente esclusi nel suo interno (Walsh 2009: 4).
\end{abstract}

L'interculturalismo funzionale, in altre parole, è una risposta agli interessi ed alle necessità delle istituzioni sociali dominanti. Bisogna ricordare che la quasi totalità delle azioni implementate dalle associazioni che abbiamo presentato sono finanziate dalle amministrazioni pubbliche: quando il finanziamento non arriva dal pubblico, proviene da qualche fondazione bancaria o istituzione religiosa. La linea di finanziamento ed il tipo di progetti a cui si concedono questi finanziamenti, riflettono le prospettive dell'ente finanziatore. Di fatto, osservando il dibattito teorico e le prassi operative in ambito politico, economico, associativo e, di conseguenza nel modo in cui l'argomento viene trattato dalla comunicazione di massa, il tema dell'interculturalismo è declinato in maniera pressoché univoca. Nella misura in cui le associazioni restano imbrigliate in un approccio interculturale isolato (e non come un principio trasversale), ed attaccate ai meccanismi di finanziamento proposti dal sistema, è abbastanza improbabile che si veda orientare in un senso diverso il dibattito sull'interculturalità.

Come abbiamo voluto dimostrare, il tema dell'interculturalismo in Spagna è strettamente connesso con il tema della migrazione e della diversità (nazionale più che culturale) che ne sembra conseguire.

Solo timidamente si lega alla diversità culturale ed etnica, rappresentata quasi esclusivamente dalla popolazione gitana. La "cultura gitana", nonostante sia stata trattata come un'entità statica, come le altre culture nazionali, non ha generato negli anni la necessità di discorsi e pratiche interculturali. Catalani.

Lo stesso esempio potrebbe essere applicato alle minoranze nazionali del paese, come per esempio Baschi e

In altri termini, mantenere o no un programma d'immersione linguistica o di normalizzazione linguistica in una comunità autonoma della Spagna (in quelle aree in cui convivono per lo meno due lingue ufficiali) non sarà mai interpretato in chiave interculturale, mentre un programma che cerchi di disciplinare le modalità didattiche della lingua ad uno studente di "nuovo ingresso" che non la conosca e che sia di un'altra nazionalità sarà inteso certamente secondo tale prospettiva.

Abbiamo ripetuto più volte che le normative, le politiche ed i dibattiti parlamentari, nel fare riferimento al tema dell'interculturalismo non fanno che riferirsi all'immigrazione, ai migranti ed alle loro culture. Estremizzando, si potrebbe sostituire il termine "inclusione" con termini espressamente legati all'immigrazione, e si vedrebbe che i discorsi, mantenendo pienamente il loro senso, sarebbero anche più attinenti con le pratiche: così "educazione interculturale" sarebbe "educazione per immigrati" o "convivenza interculturale" sarebbe "convivenza in contesti di immigrazione".

Sembrerebbe dunque che l'introduzione di questi concetti nei vocabolari politici, scolastici e mediatici possa rappresentare una strategia per le associazioni che si occupano degli immigrati, che nella pratica nascondono spesso chiare dimensioni assimilazioniste dove "Ioro" devono valorizzare ed apprendere "la nostra cultura", "loro" devono rispettare la "nostra" cultura ed integrarsi, giacché "noi" li stiamo aiutando essendo "interculturali".

In definitiva a fronte di un ampio ed appassionante dibattito sul lessico e sulla forma, sostanzialmente permangono delle logiche fortemente identitarie in ragione delle quali si rende necessaria l'apertura di una discussione preliminare sull'idea di inclusione sociale nelle società occidentali globalizzate. Ciò al fine di avviare un percorso che riconosca la piena dignità alle espressioni culturali altre dalle autoctone, foriere non sempre (e non solo) di attriti e di difficoltà, ma spesso anche di motivi di profondo arricchimento.

\title{
References
}

Agrela Romero, B. (2006). Análisis antropológico de las políticas sociales dirigidas a la población inmigrante. Tesi di dottorato difesa presso il Departamento de Trabajo Social y Servicios Sociales dell' Università di Granada.

Andreo Tudela, J.C. (2004). Políticas multiculturalistas y programas de orientación multiculturalista. In Suplementos OFRIM No 11. Madrid: Dirección General de Inmigración. Consejería de Familia y Asuntos Sociales. Comunidad de Madrid. 
Andreo Tudela, J.C., \& Santa Cruz Álvarez, S.A. (2011). Gestión de la extranjería y la inmigración en el marco comparado de Andalucía, Lombardía y Valonia. Testo presentato in occasione del I Congreso Internacional de Migración en Andalucía. Granada: Instituto de Migraciones, Universidad de Granada.

Barrett, M. (2013). Interculturalism and multiculturalism: Concepts and controversies. In M. Barrett (Ed.), Interculturalism and Multiculturalism: Similarities and Differences (pp.15-41). Strasbourg: Council of Europe Publishing.

Cárdenas Rodríguez, R. 2002. Interculturalidad e inmigración: medidas para favorecer la integración. Cuestiones pedagógicas: Revista de Ciencias de la Educación, 16, 119-138.

Cruz Roja. (2006). Pistas metodológicas para la sensibilización intercultural. Madrid, Ministerio de trabajo y asuntos sociales.

De Lucas, J. (2002). La estigmatización del multiculturalismo. Seis falacias. Temas para el debate, 89, 19-22.

Diaz, R. \& Alonso, G. (1998). Integración e interculturalidad en épocas de globalización. Presentazione al Primer Congreso Virtual de Antropología y Arqueología.

Dietz, G. (2000). El desafío de la interculturalidad. El voluntariado y las ONG ante el reto de la inmigración. El caso de la ciudad de Granada. Granada: Proyecto Sur de Ediciones/Laboratorio de Estudios Interculturales.

García Castaño, F.J., Pulido, Moyano, R. \& Montes del Castillo, A. (1999). La educación multicultural y el concepto de cultura. In García Castaño, F.J. \& Granado Martínez, A. (Eds.), Lecturas para educación intercultural (pp.47-80). Madrid: Trotta.

García Castaño, F.J., \& Granados Martinez, A. (2002). ¿Qué hay de intercultural en las acciones interculturales? (discursos, programas, educación, diseños de intervención...). El caso de la atención a los inmigrantes extranjeros. Ponencia presentada al II Congreso sobre la inmigración en España. Madrid: Universidad de Comillas, Instituto Universitario Ortega y Gasset.

García Castaño, F.J., Fernández Echevarría, J., Rubio Gómez, M. \& Soto Páez, M.L. (2007). Inmigración extranjera y educación en España: algunas reflexiones sobre el "alumnado de nueva incorporación. In M.A. Alegre \& J. Subirats (Eds.), Educación e inmigración: nuevos retos para España en perspectiva comparada (pp. 309-359). Madrid: Centro de Investigaciones Sociológicas.

García Castaño, F.J., \& Olmos Alcaraz, A. (2010). La promoción de la plena integración de los extranjeros por parte de las administraciones públicas: ¿una protección legal para la construcción de una sociedad multicultural?. In Monereo Pérez, J.L. (Dir.). Protección jurídico-social de los trabajadores extranjeros (pp.111-117). Granada: Comares.

García Castaño, F.J., Olmos Alcaraz, A., Contini, P., \& Rubio Gómez, M. (2011). Criticas y superaciones conceptuales en la gestión de la diversidad cultural. In E. Gualda Caballero. (Eds.), Inmigración, ciudadanía y gestión de la diversidad (pp.31-65). Sevilla: Universidad Internacional de Andalucía.

García Castaño, F.J., Olmos Alcaraz, A., \& Contini, P. (2012). La gestión de la diversidad cultural en Andalucía: cuando hablar de interculturalidad es hablar de inmigración. In J.L. Monereo Pérez, F.J. García Castaño \& J.A. Fernández Avilés (Eds.), La inmigración en Andalucía. Instituciones, aspectos jurídicos-sociales y culturales (pp. 599-618). Granada: Comares.

García Cano-Torrico, M., Márquez Lepe, E., González Barea, B., \& Agrela Romero, B. (2009). Nociones y prácticas en torno a la interculturalidad: el alumnado inmigrante, catalizador de la(s) diversidad(es) en los centros educativos. In N. Palaiologou (Ed.), Intercultural Education: Paideia, Polity, DemoI). Cd-rom Proceedings of the International Conference co-organized by the International Association for Intercultural Education (IAIE) and the Hellenic Migration Policy Institute (IMEPO), under the aegis of Unesco.

Giménez Romero, C. (2003). Pluralismo, Multiculturalismo e Interculturalidad. Propuesta de clarificación y apuntes educativos. Educación y Futuro digital: Educación Intercultural, 8.

Herrera, J.M. (2000) Caritas y la inmigración. Documentación social, 121, 253-270.

López Salas, A.M. (2005). Inmigrantes y Estados: la repuesta política ante la cuestión migratoria. Barcelona: Anthropos.

Leunda, J. (2008). Integración e interculturalidad. REMHU. Revista Interdisciplinar da Mobilidade Humana, 31, 263-276.

Márquez Lepe, E. (2006). La gestión política de la diversidad cultural en España: análisis de los discursos parlamentarios sobre inmigración. Tesi di Dottorato difesa presso il Departamento de Antropología Social de la Universidad de Granada.

Martínez Chicón, R. (2012). Los planes integrales para la inmigración en Andalucía: revisión histórico-crítica y significación del actual. In J.L. Monereo, F.J. García \& J.A. Fernández (Dir.), La inmigración en Andalucía. Instituciones, aspectos jurídico-sociales y culturales (pp.201-212). Granada: Comares.

Meer, N. \& Modood, T. (2013). Interacting interculturalism with multiculturalism: observations on theory and practice. In M. Barrett et al. (Eds.), Interculturalism and multiculturalism: similarities and differences (pp.111-131). Strasbourg: Council of Europe Publishing.

Olmos Alcaraz, A. (2004). Educación Intercultural en Andalucía: normativa, discurso político y práctica. Presentazione in occasione del Congreso Internacional de Educación Intercultural: formación del profesorado y práctica escolar, Madrid, 15-17 marzo 2004. [Online] Available: http://www.uned.es/congreso-inter-educacion-intercultural/Grupo_discusion_4/17. A.pdf (Agosto 20, 2015).

Olmos Alcaraz, A. (2007). La inmigración extranjera en el discurso político de Andalucía: un análisis del debate parlamentario. In R. Zapata-Barrero \& T.A. Van Dijk (Eds.), Discursos Políticos y Sociales sobre la inmigración en España: los medios de comunicación, los parlamentos y las administraciones (pp.131-157). Barcelona, Fundación CIDOB.

Olmos Alcaraz, A. (2008). Estudiar migraciones desde la Antropología Social: una investigación sobre la construcción social de la alteridad en contextos educativos. Migraciones, 23, 151-171.

Olmos Alcaraz, A. (2009). La población inmigrante extrajera y la construcción de la diferencia: discursos de alteridad en el sistema educativo andaluz, Tesi di Dottorato difesa presso il Departamento de Antropología Social, Universidad de Granada.

Olmos Alcaraz, A. (2010). Construcción discursiva del alumno inmigrante extranjero en Andalucía: el otro en nuestras escuelas. Revista de Educación, 353, 469-493. 
Pulido Moyano, R. (2005). Sobre el significado y los usos de los conceptos de interculturalidad y multiculturalidad. In T. Fernández \& J. G. Molina (Coords.), Multiculturalidad y educación (pp. 19-35). Madrid, Alianza.

Rodríguez García, J.A. 2011. La integración intercultural en España: El mestizaje constitucional democrático. Migraciones Internacionales, 6 (2), 193-222.

Santos, B. De Sousa (2011). Introducción: las epistemologías del sur. In CIDOB (org.), Formas-Otras. Saber, nombrar, narrar, hacer (pp. 9-22). Barcelona: CIDOB Ediciones.

Santamaría, E. (2002). La incógnita del extraño. Barcelona: Anthropos.

Tubino, F. (2004). Del interculturalismo funcional al interculturalismo crítico. Rostros y fronteras de la identidad, 151.

Walsh, C. (2009). Interculturalidad y (de)colonialidad. Perspectivas críticas y políticas. Presentazione al XII Congreso ARIC, Florianópolis, Brasil, 29 de junio de 2009.

Walsh, C. (2010). Interculturalidad crítica y pedagogía de-colonial: In-surgir, re-existir y re-vivir. Entrepalabras, Revista de Educación en el Lenguaje, la Literatura y la Oralidad, 3-4.

Zanfrini L. (2004), Sociologia della convivenza interétnica. Roma: Laterza. 